INJE-TP-01-06, hep-th/0107065

\title{
Quintessence and Brane world scenarios
}

\author{
Y.S. Myung* \\ Department of Physics, Graduate School, Inje University, Kimhae 621-749, Korea
}

\begin{abstract}
We discuss the possibility of quintessence in the dilatonic domain walls including the Randall-Sundrum brane world. We obtain the zero mode effective action for gravitating objects in the dilatonic domain wall. First we consider the four dimensional (4D) gravity and the Brans-Dicke graviscalar with a potential. This can be further rewritten as a minimally coupled scalar with the Liouville-type potential in the Einstein frame. However this model fails to induce the quintessence on the dilatonic domain wall because the potential is negative. Second we consider the $4 \mathrm{D}$ gravity with the dilaton. In this case we find also a negative potential. Any negative potential gives us negative energy density and positive pressure, which does not lead to an accelerating universe. Consequently it turns out that the zero mode approach of the dilatonic domain wall cannot accommodate the quintessence in cosmology.
\end{abstract}

Compiled at December 25, 2018: 218.

*Email-address : ysmyung@physics.inje.ac.kr 


\section{INTRODUCTION}

Recently cosmic quintessence has proposed to be an alternative way to understand the astronomical data of supernova which indicate that the universe is presently accelerating [1 3]. Also this may be considered as a method to resolve the cosmological constant problem. This is possible because instead of the fine-tuning, it provides a model of slowly decaying cosmological constant. Several literature discussed on this issue within the string theory [4.5] and cosmological context [6,7].

More recently authors in ref. [8] also considered this problem in the dilatonic domain wall. After the integration over the dual holographic field theory, they obtained a dilatonic gravity with the potential on the brane. They used the Hamilton-Jacobi method inspired by the holographic renormalization group to investigate the intrinsic Friedmann-RobertsonWalker (FRW) cosmology on the brane. It was shown that the holographic quintessence is allowed on the dilatonic brane because a Liouville-type potential appears on the brane. This case requires a negative slowly varying bulk potential which implies that the bulk space is a five dimensional anti de Sitter $\left(\mathrm{AdS}_{5}\right)$ spacetime.

In this paper we wish to deal with the same issue within a different context. An idea of the brane world scenario is that our universe may be a brane embedded in the higher dimensional space 9 13. A concrete model is a single 3-brane embedded in the $\mathrm{AdS}_{5}$ space [12]. Randall and Sundrum (RS) have shown that a longitudinal part $\left(h_{\mu \nu}\right)$ of the metric fluctuations satisfies the Schrödinger-like equation with an attractive delta-function. As a result, the massless Kaluza-Klein (KK) modes which describe the localized gravity on the brane were found. Furthermore, the massive KK modes which reside in the extra dimension lead to corrections to the Newtonian potential. We would like to point out that this has been done in the 4D Minkowski brane with the RS gauge. It seems that this gauge is so restrictive. In order to have an interesting cosmological model on the brane, we may include the graviscalar (KK scalar) at the beginning [14 -16].

In this work we will not use the holographic renormalization group to find the brane potential. Instead we follow an idea for the genuine brane world scenario such that the universe is filled with the zero modes of the bulk fields which are trapped on the brane by the gravitational interaction [17]. In our approach the relevant scalar is either the dilaton $(d)$ or the graviscalar $(h)$. The condition that the zero mode is localized on the brane corresponds to the normalizability of the ground state wave function on the brane. Hence we have to find the finite zero-mode action of the dilatonic domain wall to study its cosmological implication. Fortunately we can obtain the finite action with the Liouville-type potentials. But we fail to find any accelerating universe from the dilatonic domain wall.

The organization of our paper is as follows. In Sec. II we briefly review a simple model for the quintessence. We derive the $4 \mathrm{D}$ effective action on the brane by using the zero-mode approach in Sec. III. In Sec. IV we consider the graviscalar as a dynamic scalar on the brane. And we discuss its role for cosmological implication. In Sec. V we consider the

\footnotetext{
${ }^{1}$ In fact, this gauge for the 5D metric fluctuation $h_{M N}$ is composed of Gaussian-normal gauge $\left(h_{44}=h_{4 \mu}=0\right)$ and $4 \mathrm{D}$ transverse, traceless gauge $\left(\partial^{\mu} h_{\mu \nu}=0, h_{\mu}^{\mu}=0\right)$.
} 
dilaton as a dynamic scalar for deriving a cosmological evolution. Finally we discuss our results in Sec. VI.

\section{QUINTESSENCE}

Quintessence endeavors to handle a cosmological problem with a dynamic negative pressure. A minimally coupled scalar field with a potential that decreases as the field increases is usually introduced for this purpose [6]. The action is given by

$$
S_{\mathrm{Q}}=\frac{1}{2 \kappa_{4}^{2}} \int d^{4} x \sqrt{-g}\left[R-(\partial \phi)^{2}-2 V(\phi)\right]
$$

with the $4 \mathrm{D}$ gravitational constant $\kappa_{4}^{2}$. This is a canonically normalized scalar action coupled to the $4 \mathrm{D}$ gravity. The Einstein equation is

$$
R_{\mu \nu}-\frac{1}{2} R g_{\mu \nu}=T_{\mu \nu}
$$

with

$$
T_{\mu \nu}=\partial_{\mu} \phi \partial_{\nu} \phi-\frac{1}{2}(\partial \phi)^{2} g_{\mu \nu}-V(\phi) g_{\mu \nu}
$$

Considering the FRW flat metric of $d s_{F R W}^{2}=-d t^{2}+\mathcal{R}^{2}(t) d x^{i} d x_{i}$, the equation of motion for $\phi$ and the conservation law of $\nabla_{\mu} T^{\mu 0}=0$ lead to the the same equation as $\ddot{\phi}+3 \frac{\dot{\mathcal{R}}}{\mathcal{R}} \dot{\phi}+V^{\prime}(\phi)=$ 0. The two FRW equations are given by

$$
\frac{\dot{\mathcal{R}}^{2}}{\mathcal{R}^{2}}=\frac{\rho}{3}, \quad \frac{\ddot{\mathcal{R}}}{\mathcal{R}}=-\frac{\rho+3 p}{6} .
$$

Assuming $\phi=\phi(t)$ for cosmological purpose, then the energy density and pressure are given by

$$
\rho=\frac{1}{2} \dot{\phi}^{2}+V(\phi), \quad p=\frac{1}{2} \dot{\phi}^{2}-V(\phi)
$$

The corresponding equation of state takes the form

$$
\omega \equiv \frac{p}{\rho}=\frac{\dot{\phi}^{2}-2 V(\phi)}{\dot{\phi}^{2}+2 V(\phi)} .
$$

The equation of state ranges over $-1<\omega<1$, depending on the dynamics of the field. We note that when $\dot{\phi}^{2}<V(\phi)$ on later time, an accelerating universe appears from the second equation in Eq.(4). In this model $\phi$ and $\rho$ scale as [6]

$$
\frac{\partial \phi}{\partial \mathcal{R}}=\frac{\sqrt{3(1+\omega)}}{\mathcal{R}}, \quad \rho \sim \frac{1}{\mathcal{R}^{3(1+\omega)}} .
$$

Using the relation of $V(\phi)=(1-\omega) \rho / 2$ together with $\phi=\sqrt{3(1+\omega)} \ln \mathcal{R}$, the positive potential takes the form 


$$
V_{\text {quint }}(\phi)=V_{0} e^{-\sqrt{3(1+\omega)} \phi}, \quad 3(1+\omega)<2
$$

which is a kind of Liouville-type potential that decreases as $\phi$ increases. According to the theory of quintessence, the dark energy of the universe is dominated by the scalar potential which is still rolling to its minimum of $V_{\text {quint }}=0$. We require its minimum at $\phi=\infty$ conventionally. The above potential is suited well for the quintessence. For example, if one takes $\omega=-1 / 2<-1 / 3, V_{\text {quint }}(\phi)=V_{0} e^{-\sqrt{3 / 2} \phi}$ can induce an accelerating universe. In the next section we wish to check whether or not the above-type potential can be found from the dilatonic domain wall model.

\section{4D EFFECTIVE ACTION ON THE BRANE}

We start with the 5D bulk action and 4D domain wall action as [18,19.

$$
S=S_{\text {bulk }}+S_{\mathrm{DW}}
$$

with

$$
\begin{aligned}
S_{\text {bulk }} & =\frac{1}{2 \kappa_{5}^{2}} \int d^{5} x \sqrt{-G}\left[R_{5}-\frac{4}{3} \partial_{M} D \partial^{M} D-e^{-2 a D} \Lambda\right], \\
S_{D W} & =-\sigma_{\text {DW }} \int d^{4} x \sqrt{-\gamma} e^{-a D},
\end{aligned}
$$

where $\sigma_{\mathrm{DW}}$ is the tension of the domain wall and $\gamma$ is the determinant of the induced metric $\gamma_{\mu \nu}=\partial_{\mu} X^{M} \partial_{\nu} X^{N} G_{M N}$ on the domain wall. Here $M, N=0,1,2,3,4\left(x^{4}=z\right)$ and $\mu, \nu=0,1,2,3\left(x^{\mu}=x\right)$. " $D$ " denotes the dilaton. We are interested in the dilatonic domain wall solution with

$$
\bar{G}_{M N}=H^{-2}(z) \eta_{M N}, \quad e^{2 \bar{D}}=[H(z)]^{\frac{9 a}{4}}, \quad \Lambda=\frac{32 k^{2}}{\Delta}, \quad \sigma_{\mathrm{DW}}=\frac{16 k}{|\Delta| \kappa_{5}^{2}}
$$

with the conformal factor $H(z)=\left(1+4 k \frac{\Delta+2}{\Delta}|z|\right)^{4 /(3 \Delta+6)}, \Delta=-\frac{8}{3}+\frac{3 a^{2}}{2}$ and $\eta_{M N}=\operatorname{diag}[-+$ +++ . Here overbar $\left(^{-}\right)$means the vacuum solution. Also we choose a negative bulk cosmological constant $\Lambda<0$ with $\Delta<0$ and a positive domain wall tension $\sigma_{\mathrm{DW}}>0$ for the fine-tuning. The condition of $\Delta<0$ leads to the constraint on $a: 0<a^{2}<16 / 9$.

In order to obtain the zero mode effective action, we propose the metric $G_{M N}=$ $H^{-2}(z) \hat{G}_{M N}$ where $\hat{G}_{M N}$ is a function of " $x$ " only. Explicitly the line element is given by

$$
\begin{aligned}
d S_{5}^{2} & =G_{M N} d x^{M} d x^{N}=H^{-2} \hat{G}_{M N} d x^{M} d x^{N} \\
& =H^{-2}\left[g_{\mu \nu}(x) d x^{\mu} d x^{\nu}+h(x)^{2} d z^{2}\right]
\end{aligned}
$$

Further we introduce the dilaton $d(x)$ in the form of

\footnotetext{
${ }^{2}$ For $a=0$ case, this reduces to the second RS vacuum solution exactly 20].
} 


$$
e^{2 D}=[H(z)]^{\frac{9 a}{4}} e^{2 d(x)}
$$

Hereafter the graviton $g_{\mu \nu}(x)$, graviscalar $h(x)$, and dilaton $d(x)$ play the role of zero modes. Off-diagonal elements are not included because they are not necessary here 5 . $h(x)$ is related to the radion that is necessary for stabilizing the distance between two branes in the first RS model [11]. Substituting Eq.(13) together with Eq.(14) into (9) and then integrating it over $z$ lead to the $4 \mathrm{D}$ effective action

$$
S_{\mathrm{DDW} 1}=\frac{1}{2 \kappa_{5}^{2}} \int d^{4} x \sqrt{-g}\left[-\frac{\Delta}{2 k(\Delta+4)}\left(h R-\frac{4}{3} h(\partial d)^{2}\right)-\frac{16 k}{\Delta}\left(h e^{-2 a d}+\frac{1}{h}-2 e^{-a d}\right)\right] .
$$

If we define the $4 \mathrm{D}$ gravitational constant as

$$
\kappa_{4}^{2}=-2 k \frac{\Delta+4}{\Delta} \kappa_{5}^{2},
$$

then the above action leads to

$$
S_{\mathrm{DDW} 2}=\frac{1}{2 \kappa_{4}^{2}} \int d^{4} x \sqrt{-g}\left[h R-\frac{4}{3} h(\partial d)^{2}-\frac{32 k^{2}(\Delta+4)}{\Delta^{2}}\left(2 e^{-a d}-h e^{-2 a d}-\frac{1}{h}\right)\right] .
$$

Here $\kappa_{4}^{2}>0$ is automatically guaranteed because we choose $\Delta<0$ and $\Delta+4>0$. If $\Delta>0$, the $4 \mathrm{D}$ gravity is not trapped on the dilatonic domain wall [22]. This action is very important for our study. We note here that the limit of $k \rightarrow 0$ means that $\mathrm{AdS}_{5}$ space $\rightarrow$ $5 \mathrm{D}$ Minkowski space, and tension domain wall $\rightarrow$ tensionless domain wall. In this limit we recover the conventional KK model without the last potential in Eq.(17).

\section{BRANS-DICKE GRAVISCALAR AS A DYNAMIC SCALAR}

Since the above action has two different scalars with the mixed potential, we first consider the dynamic graviscalar $(d(x)=0, h(x) \neq 1)$. In the next section we will deal with the dilaton. The action (17) reduces to the Brans-Dicke (BD) model with a different potential 18

$$
S_{\mathrm{BD}}=\frac{1}{2 \kappa_{4}^{2}} \int d^{4} x \sqrt{-g}\left[h R-\frac{32 k^{2}(\Delta+4)}{\Delta^{2}}\left(2-h-\frac{1}{h}\right)\right] .
$$

The first term $(h R)$ is the $\mathrm{BD}$ term when the BD parameter $w=0$ [23]. This comprises the massless Kaluza-Klein modes $g_{\mu \nu}, g_{44}\left(\sim h^{2}\right)$ but with $g_{\mu 4}\left(\sim A_{\mu}\right)=0$ 帛. In this sense, we wish to call $h$ the BD scalar. The second term arises from the facts : a dilatonic domain

\footnotetext{
${ }^{3}$ One may introduce off-diagonal term of $2 A_{\mu}(x) d x^{\mu} d z$ for general discussion 21].

${ }^{4}$ This model is equivalent to $S_{\mathrm{KK}}=\frac{1}{2 \kappa_{5}^{2}} \int d^{5} x \sqrt{-G} R_{5}$ with a factorizable geometry of $H=1(k=$ $0)$.
} 
wall is located at $z=0$ ("2"), the bulk spacetime is an $\mathrm{AdS}_{5}$ space (" $h$ "), and $h$ is the KK scalar(" $1 / h ")$. Equivalently, this means that the domain wall configuration describes non-factorizable geometry with a conformal factor $H(z)$. Especially for $a=0$ case, we have the effective action for the RS-type model [20]: $\mathcal{L}_{\mathrm{RS}}=\sqrt{-g}\left[h R-6 k^{2}(2-h+1 / h)\right] / 2 \kappa_{4}^{2}$.

In order to obtain a canonical scalar action, we use a conformal transform : $g_{\mu \nu} \rightarrow \Omega^{-2} \bar{g}_{\mu \nu}$ with $\Omega^{2}=h$. This implies that we move from the string-like frame to the Einstein frame. The resulting action takes the form

$$
S_{\mathrm{BD} 1}=\frac{1}{2 \kappa_{4}^{2}} \int d^{4} x \sqrt{-\bar{g}}\left[R-\frac{3}{2} \frac{(\partial h)^{2}}{h^{2}}-\frac{32 k^{2}(\Delta+4)}{\Delta^{2}} \frac{1}{h^{2}}(2-h-1 / h)\right] .
$$

Let us define $h \equiv e^{\sqrt{2 / 3} \Phi}$ to obtain a canonical form like (四). Then we have a desired action

$$
S_{\mathrm{BD} 2}=\frac{1}{2 \kappa_{4}^{2}} \int d^{4} x \sqrt{-\bar{g}}\left[R-(\partial \Phi)^{2}-\frac{32 k^{2}(\Delta+4)}{\Delta^{2}}\left(2 e^{-\sqrt{8 / 3} \Phi}-e^{-\sqrt{6} \Phi}-e^{-\sqrt{2 / 3} \Phi}\right)\right]
$$

which implies the graviscalar potential on the domain wall

$$
V_{G S}(\Phi)=\frac{16 k^{2}(\Delta+4)}{\Delta^{2}}\left(2 e^{-\sqrt{8 / 3} \Phi}-e^{-\sqrt{6} \Phi}-e^{-\sqrt{2 / 3} \Phi}\right) .
$$

All of terms belong to the Liouville-type potential. The only last term that comes from the genuine graviscalar satisfies a criterion of the quintessence $(\sqrt{2 / 3}<\sqrt{2})$. Unfortunately it belongs to a negative potential. For this purpose we rewrite the potential as

$$
V_{G S}(\Phi)=-\frac{16 k^{2}(\Delta+4)}{\Delta^{2}} e^{-\sqrt{6} \Phi}\left(e^{\sqrt{2 / 3} \Phi}-1\right)^{2}
$$

which shows obviously that the graviscalar potential is always negative in the whole value of $\Phi$. Explicitly, the potential starts with $V_{G S}=0$ at $\Phi=0$, decreases until it arrives at the minimum and then again increases as $\Phi$ increases. And it takes the final form of $V_{G S}(\infty) \rightarrow 0$. Hence the graviscalar potential on the dilatonic domain wall is basically different from the single Liouville-type potential of Eq.(8), which decreases monotonically as a scalar increases. For the special case of $a=0$ (non-dilatonic brane), we find the RS-type potential on the brane

$$
V_{R S}(\Phi)=-3 k^{2} e^{-\sqrt{6} \Phi}\left(e^{\sqrt{2 / 3} \Phi}-1\right)^{2}
$$

which is also negative. This leads to the RS vacuum solution $V_{R S}=0$ only for the purely graviton propagation with $\Phi=0$. Even if we consider the dilatonic domain wall $(a \neq 0)$, this does not change the negative nature of potential. Here we have always a negative energy density $\rho=\dot{\Phi}^{2} / 2+V_{G S}(\Phi)$ and a positive pressure $p=\dot{\Phi}^{2} / 2-V_{G S}(\Phi)$. This contrasts to the quintessence which is based on the positive energy density and negative pressure with $p<-\rho / 3$. Hence even though we obtain the Liouville-type potentials, we cannot find any accelerating universe from the the dilatonic domain wall including the RS-brane world scenario. This means that the KK scalar is not suitable for describing the quintessence in cosmology. 


\section{DILATON AS A DYNAMIC SCALAR}

In this section we study the dilatonic case $(d(x) \neq 0, h(x)=1)$ because we fail to obtain an appropriate potential for the quintessence using the graviscalar. From Eq.(17) one has

$$
S_{\text {dil }}=\frac{1}{2 \kappa_{4}^{2}} \int d^{4} x \sqrt{-g}\left[R-\frac{4}{3}(\partial d)^{2}-\frac{32 k^{2}(\Delta+4)}{\Delta^{2}}\left(2 e^{-a d}-e^{-2 a d}-1\right)\right] .
$$

Introducing $\tilde{d}(x)=\sqrt{3 / 4} d(x)$, the above action leads to

$$
S_{\text {dil1 }}=\frac{1}{2 \kappa_{4}^{2}} \int d^{4} x \sqrt{-g}\left[R-(\partial \tilde{d})^{2}-\frac{32 k^{2}(\Delta+4)}{\Delta^{2}}\left(2 e^{-a \sqrt{4 / 3} \tilde{d}}-e^{-2 a \sqrt{4 / 3} \tilde{d}}-1\right)\right] .
$$

Here we can read off its potential

$$
V_{d i l}(\tilde{d})=\frac{16 k^{2}(\Delta+4)}{\Delta^{2}}\left(2 e^{-a \sqrt{4 / 3} \tilde{d}}-e^{-2 a \sqrt{4 / 3} \tilde{d}}-1\right)
$$

We point out that the first term comes from the tension of the domain wall $\left(\sigma_{D W} e^{-a D}\right)$ and the second from the bulk potential term $\left(\Lambda e^{-2 a D}\right)$. The last term arises from the ansatz for the zero modes Eq.(13) with $h=1$. Further this can be expressed as

$$
V_{d i l}(\tilde{d})=-\frac{16 k^{2}(\Delta+4)}{\Delta^{2}}\left(e^{-a \sqrt{4 / 3} \tilde{d}}-1\right)^{2} .
$$

It is noted that $V_{d i l}(\tilde{d})$ decreases monotonically until it arrives the minimum of $V_{d i l}(\infty)=-1$ as $\tilde{d}$ increases. But this belongs to a negative potential as is obviously shown by (27). Hence we find that the dilaton cannot induce the quintessence.

\section{DISCUSSIONS}

We investigate the zero mode sector to the 5D dilatonic domain wall solution for cosmological purpose. First we study the 4D gravity with the graviscalar on the brane. Assuming the FRW metric on the brane, one has a minimally coupled graviscalar with the potential. Although the graviscalar potential belongs to a kind of Liouville-type potential, it remains negative in the whole value of $\Phi$. This gives us in turn the negative energy density and positive pressure, which contrasts to the usual quintessence that has the positive energy density and negative pressure. Second we consider the $4 \mathrm{D}$ gravity with the dilaton. Also we find a negative dilatonic potential. Hence we cannot find the quintessence by using either the graviscalar or the dilaton. Furthermore the mixed potential from Eq.(17) takes a form of $\sim-h^{-3}\left(h e^{-a d}-1\right)^{2}$, which is negatively definite. That is, even if we include both the graviscalar and dilaton, we find a negative potential. All of potentials which we find here belong to the negative unstable potential. As a by-product, this implies that the RS vacuum solution of $d=0, \Phi=0(h=1)$ may not be a truly vacuum solution except the RS gauge. In the limit of $k \rightarrow 0$, we find the equation of state $p=\rho$ with $\omega=1$ which behaves as a stiff matter with $\rho=p \sim a^{-6}$ for both the graviscalar and dilaton cases . 
Finally we wish to comment on the result for the holographic quintessence [8]. In that case they used the Hamilton-Jacobi equation to obtain a single dilatonic potential $U(\phi)=$ $e^{b\left(\phi-\phi_{0}\right)}$ with $b<0$ on the brane. Actually this corresponds to the first term of Eq.(26) that comes just from the tension of the domain wall $\left(\sigma_{D W} e^{-a D}\right)$. Of course, a single term like this can induce an accelerating universe. But we obtain three terms which give totally us the dilatonic potential Eq.(26). So it seems to exist a difference between our zero-mode approach and holographic approach.

\section{ACKNOWLEDGEMENT}

We thank to C. R. Cai and G. Kang for helpful discussions. This work was supported in part by the Brain Korea 21 Program of Ministry of Education, Project No. D-1123 and KOSEF, Project No. 2000-1-11200-001-3. 


\section{REFERENCES}

[1] S. Perlmutter et al.(Supernova Cosmology Project), "Measurements of the Cosmological Parameters Omega and Lambda from the First 7 Supernovae at z $>=0.35$ ", Astrophys. J. 483, 565(1997), astro-ph/9608192.

[2] R. R. Caldwell, R. Dave, and P. J. Steinhard, "Cosmological Imprint of an Energy Component with General Equation of State", Phys. Rev. Lett. 80, 1582(1998), astroph/9708069.

[3] P. M. Garnavich et al., "Supernova Limits on the Cosmic Equation of State", Astrophys. J. 509, 74(1998), astro-ph/9806396.

[4] E.Witten, "Quantum Gravity in de Sitter Space", hep-th/0106109.

[5] S. Hellerman, N. Kaloper, and L. Susskind, "String Theory and Quintessence", hepth/0104180; W. Fischler, A. Kashani-Poor, R. McNees, and S. Paban, "The Acceleration of the Universe, a Challenge for String Theory", hep-th/0104181.

[6] B. Ratra and P. J. E. Peebles, "Cosmological Consequences of a Rolling Homogeneous Scalar Field", Phys. Rev. D37, 3406(1988).

[7] C. Kolda and W. Lahneiman, "Exponential Quintessence and the End of Acceleration", hep-th/0105300; M. Gasperini, "Dilatonic Interpretation of the Quintessence?", grqc/0105082; J.M.Cline, "Quintessence, Cosmological Horizons, and Self-Tuning", hepth/0105251; Ph.Brax and A.C.Davis, "Cosmological Evolution on Self-Tuned Branes and the Cosmological Constant", JHEP, 0105, 007(2001), hep-th/0104023.

[8] B. Chen and F.-L. Lin, "Holographic Cosmic Quintessence on Dilatonic Brane World", hepth/0106054.

[9] V. A. Rubakov and M. E. Shaposhnikov, Phys. Lett. B125, 136 (1983).

[10] K. Akama, Lect. Notes Phys. 176, 267(1982), hep-th/0001113.

[11] L. Randall and R. Sundrum, Phys. Rev. Lett. 83, 3370 (1999), hep-ph/9905221.

[12] L. Randall and R. Sundrum, Phys. Rev. Lett. 83, 4690 (1999), hep-th/9906064.

[13] N. Arkani-Hamed, S. Dimopoulos, G. Dvali and N. Kaloper, hep-th/9907209; W.D. Goldberger and M.B. Wise, hep-th/9907447; E. Halyo, hep-th/9909127; A. Chamblin and G.W. Gibbons, hep-th/9909130.

[14] M.G. Ivanov and I.V. Volovich, "Metric Fluctuations in Brane Worlds", hep-th/9912242.

[15] Y.S. Myung and G. Kang, "Comment on "Metric Fluctuations in Brane Worlds"", hep-th/0001003.

[16] Y.S. Myung, G. Kang and H.W. Lee, "Randall-Sandrum gauge in brane world", hepth/0001107.

[17] B. Bajc and G. Gabadadze, Phys. Lett. B474, 282(2000), "Localization of Matter and Cosmological Constant on a Brane in Anti de Sitter Space", hep-th/9912232 ; A. Pomarol, "Gauge bosons in a five-dimensional theory with localized gravity", hepth/9911294; T. Gherghetta and A. Pomarol, "Bulk Fields and Supersymmetry in a Slice of AdS", hep-ph/9911015 ; I. Oda, "Localization of Various Bulk fields on a Brane", hep-th/0009074; Y. S. Myung, "Localization of gravivector and graviscalar on the brane", hep-th/0009117; M. Mintchev and L. Pilo, "Localization of quantum fields on branes", Nucl.Phys. B592, 219(2001), hep-th/0007002.

[18] D. Youm, "A note on solutions in brane worlds", hep-th/0001166.

[19] M. Cvetic and H. H. Soleng, Phys. Rept. 282, 159( 1997), hep-th/9604090 ; H. A. Chamblin and H. S. Reall, Nucl. Phys. B562, 133 (1999), hep-th/9903225. 
[20] Y. S. Myung and H. W. Lee, "Schwarzschild black hole in the dilatonic domain wall", Phys. Rev. D63, 064034(2001), hep-th/0001211.

[21] G. Kang and Y.S. Myung, "U(1) gauge field of the Kaluza-Klein theory in the presence of branes", Phys. Rev. D63, 064036(2001), hep-th/0007197.

[22] D. Youm, "Solitons in brane worlds", hep-th/9912175.

[23] C. Brans and R.M.D. Dicke, Phys. Rev. 124, 925 (1961); R.M.D. Dicke, Phys. Rev. 125, 2163 (1962). 